\title{
Does second-line therapy affect the radiological progression of rheumatoid arthritis?
}

\author{
T. PULLAR, J. A. HUNTER, AND H. A. CAPELL \\ From the Centre for Rheumatic Diseases, University Department of Medicine, Royal Infirmary, Glasgow G31 \\ $2 E R$
}

SUMmARY The effect of 'second-line' drugs on radiological progression in rheumatoid arthritis is not clear, and previous studies have yielded contradictory results. Sixty-seven patients with rhe umatoid arthritis have been followed up clinically and radiologically for approximately 2 years (26 patients were receiving intramuscular gold, 21 penicillamine, 10 levamisole, and there were 10 controls who had consistently refused second-line therapy). Patients on gold and penicillamine showed improvement in erythrocyte sedimentation rate and haemoglobin over 2 years which was not seen in levamisole and control patients, but hand radiograph scores in all 4 groups showed statistically significant deterioration. There was a trend towards slowing of the rate of erosion in the gold and penicillamine groups in comparison with controls, but healing of erosions was extremely unusual.

It is well accepted that the use of 'second-line' drugs such as gold or penicillamine is of clinical benefit in patients with rheumatoid arthritis. In addition these drugs are known to improve laboratory indices of inflammation such as erythrocyte sedimentation rate (ESR) and haemoglobin. ${ }^{1-6}$

Few studies have been published, however, on the effect of second-line drugs on the radiological progression of the disease, and even among the reported studies there is a degree of disagreement. ${ }^{3-8}$ Carrying out such a study is fraught with difficulties, particularly in maintaining a control group over the necessary long follow-up period. Some would consider such a control group ethically unjustified; in addition we have found it impracticable to maintain these patients off second-line drugs.

We have attempted to circumvent this problem, using similar numbers of patients as in the trials of Sigler $e t a{ }^{3}{ }^{3}$ and Gibson et al., ${ }^{7}$ by following up a control group of patients who have consistently refused repeated offers of second-line therapy for at least 2 years. Indices of inflammatory disease and radiological changes in these patients have been compared with those of patients on gold, penicillamine, and levamisole.

\section{Patients and methods}

Sixty-seven patients with definite or classical Accepted for publication 8 February 1983.

Correspondence to Dr T. Pullar, Centre for Rheumatic Diseases, 35 Baird Street, Glasgow G4 0EH. rheumatoid arthritis with an active inflammatory component have been studied. No patient had previously received second-line therapy and none was concurrently receiving corticosteroids. Fifty-seven had initially been started on second-line therapy because of failure of active rheumatoid arthritis to respond to nonsteroidal anti-inflammatory drugs (NSAIDs) and other general measures and had received such therapy for approximately 2 years.

The patients on gold therapy $(n=26)$ were all receiving intramuscular sodium aurothiomalate (Myocrysin). The dose was $50 \mathrm{mg}$ weekly until clinical improvement, and thereafter the frequency was reduced to fortnightly, 3-weekly, and ultimately 4-weekly. Patients on pencillamine $(n=21)$ started on $125 \mathrm{mg}$ daily. This dose was increased by increments of $125 \mathrm{mg}$ per day at intervals of 2 to 4 weeks until a response was achieved, and the patients continued this dose thereafter. Each patient received the minimum possible dose: in 14 of the 21 patients this was more than $500 \mathrm{mg}$ daily over at least 6 months of the period of radiological monitoring. Doses of levamisole $(n=10)$ were $150 \mathrm{mg} /$ week (4 patients), $300 \mathrm{mg} /$ week (4 patients), and $450 \mathrm{mg} /$ week (2 patients). The patients were among those previously reported in a number of short-term studies. ${ }^{910}$ The control group $(n=10)$ consisted of patients who, despite clinical indications, had consistently refused second-line agents for approximately 2 years.

Hand radiographs were taken immediately before beginning second-line therapy or at the time of 
refusal of suggested treatment and again after approximately 2 years of therapy or follow-up of controls. The erythrocyte sedimentation rate and haemoglobin were measured at the start of treatment and after 2 years.

Radiographic assessment comprised 2 methods employing anteroposterior hand films scored, blind for date and patient, by 3 independent observers (H.A.C., J.A.H., T.P.). Intraobserver error was quantitated by one observer (T.P.) after an interval of 2 months.

The first method assessed defects by a modification of the method described by Sharp et al. ${ }^{11}$ Osseus defects were counted at the distal end of radius and ulna, the 5 metacarpophalangeal joints, and the 4 proximal interphalangeal joints of both hands. At each joint the number of discrete defects that could be identified were recorded to a maximum of 5 , and this score also applied in the presence of joint destruction. The sum of individual scores for all joints assessed could therefore reach a maximum of 110 . The rate of erosion before treatment was obtained by dividing the initial score by the duration of arthritis in months. Rate of erosion during treatment was assessed by dividing the difference in the initial score and final score by the time interval in months between the films.

The second chronological method of radiographic scoring comprised arranging available films in order of severity of joint damage and comparing this with the chronological order. The frequency of discrepancies in each treatment group and the control group was then compared.

The principal methods used for statistical analysis were nonparametric, but in order to compare results with other published work and calculate type II error parametric tests were also used.

\section{Results}

The patients' characteristics are shown in Table 1. In the 67 patients studied the median age was 50 years (range 24-72) and duration of disease was 6 years (range 1-25). Age, disease duration, initial ESR, and initial haemoglobin were comparable across all 4 groups (Kruskal-Wallis test, $p>0 \cdot 2$ ). Significant improvements in the ESR and haemoglobin occurred over the study period in gold and penicillamine treated patients but not in the control group or levamisole treated patients (Table 2).

The results for method 1 for the two readers T.P. and J.A.H. show a strong linear relationship with a correlation of 0.84 (Table 3 ). Since H.A.C. counted only erosions whereas the others also counted subarticular cysts, the relationship between H.A.C.'s results and the others is not linear. By separate polynomial regression H.A.C.'s results were found to correlate well with those of J.A.H. Measurement of intraobserver error showed good correlation $(r=0.926)$.

The interval between hand radiographs varied slightly (median values for control=33 months,

Table 2 Statistical significance of the change in ESR and haemoglobin over the follow-up period in treatment and control groups (Wilcoxon test)

\begin{tabular}{lllll}
\hline & Control & Gold & Penicillamine & Levamisole \\
\hline ESR & $\mathrm{p}=0.772$ & $\mathrm{p}=0.002$ & $\mathrm{p}=0.003$ & $\mathrm{p}=0.515$ \\
$\mathrm{Hb}$ & $\mathrm{p}=0.237$ & $\mathrm{p}=0.004$ & $\mathrm{p}=0.001$ & $\mathrm{p}=0.779$ \\
\hline
\end{tabular}

Table 3 Interobserver correlation

\begin{tabular}{lll}
\hline J.A.H./T.P. & J.A.H./H.A.C.* \\
\hline $\mathbf{r}$ & 0.84 & 0.92 \\
Intercept & -0.75 & -0.0054 \\
Slope & 0.97022 & 1.0036 \\
\hline
\end{tabular}

* Relationship not linear.

Table 1 Patients' characteristics

\begin{tabular}{llllll}
\hline $\begin{array}{l}\text { Treatment } \\
\text { group } M: F\end{array}$ & & $\begin{array}{l}\text { Control } \\
2: 8\end{array}$ & $\begin{array}{l}\text { Gold } \\
9: 17\end{array}$ & $\begin{array}{l}\text { Penicillamine } \\
6: 15\end{array}$ & $\begin{array}{l}\text { Levamisole } \\
2: 8\end{array}$ \\
\hline Age (yr) & Median & 52 & 57 & 53 & 47 \\
& Range & $33-65$ & $33-48$ & $33-72$ & $24-60$ \\
Duration of & Median & $4 \cdot 5$ & 5 & 8 & 6 \\
disease (yr) & Range & $1-14$ & $1-26$ & $1-20$ \\
Initial ESR & Median & 60 & 58 & 46 & 29 \\
(mm/h) & Range & $9-110$ & $5-138$ & $5-113$ & $11 \cdot 7$ \\
Initial & Median & $11 \cdot 6$ & $11 \cdot 6$ & $11 \cdot 8$ & $10 \cdot 2-14 \cdot 5$ \\
Hb (g/d) & Range & $7 \cdot 2-18 \cdot 5$ & $9 \cdot 0-15 \cdot 2$ & $8 \cdot 2-15 \cdot 4$ & 49 \\
Initial & Median & 61 & 35 & 68 & $2-89$ \\
$X$-ray score & Range & $2-73$ & $0-100$ & $0-110$ & 23 \\
Duration of & Median & 33 & 27 & 25 & $19-29$ \\
follow-up & Range & $17-54$ & $21-54$ & $19-30$ & \\
(months) & & & & & \\
\hline
\end{tabular}




\section{CONTROL}

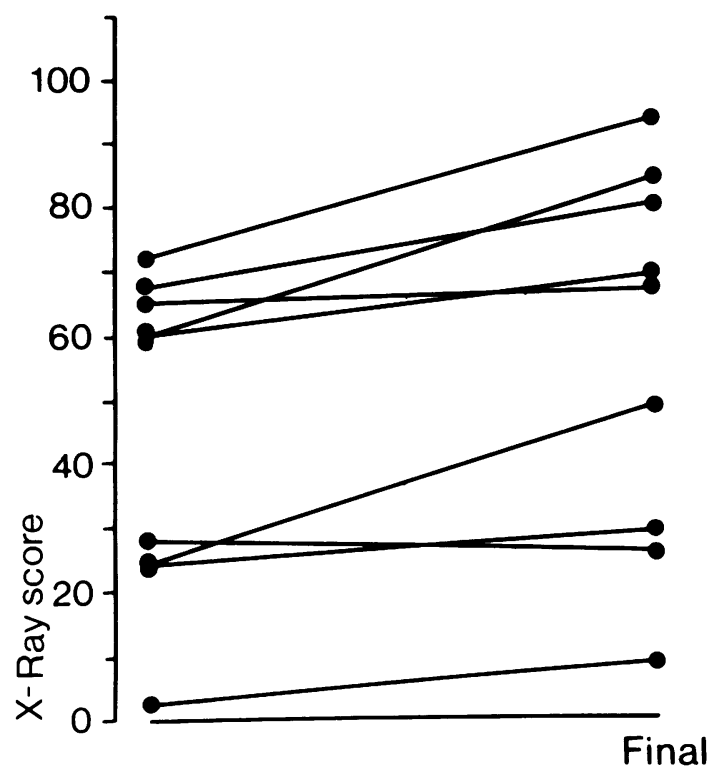

Fig. 1 Change in radiograph score in control group.

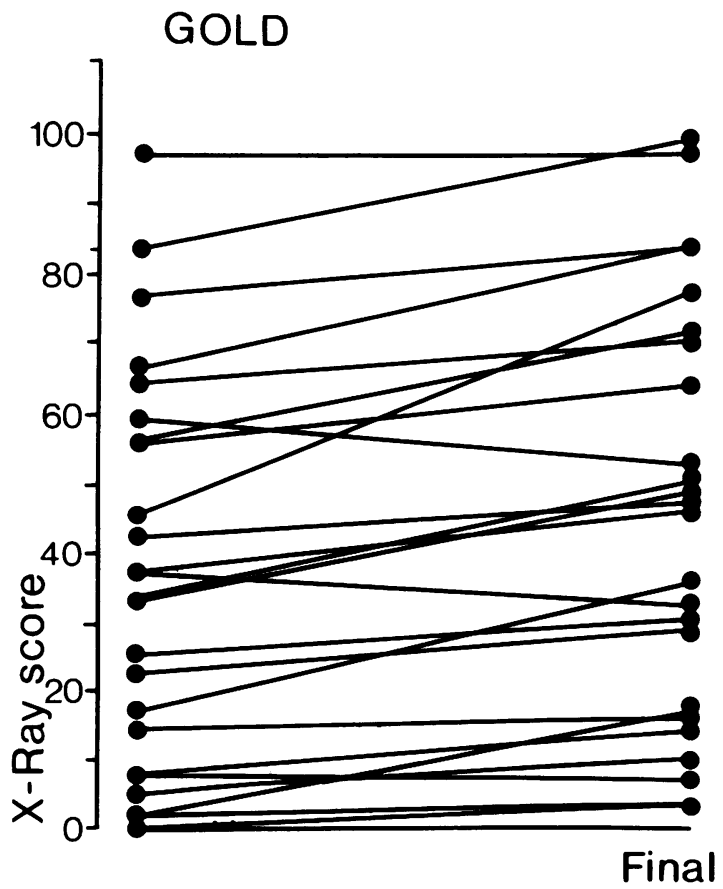

Fig. 2 Change in radiograph score in gold treated group.

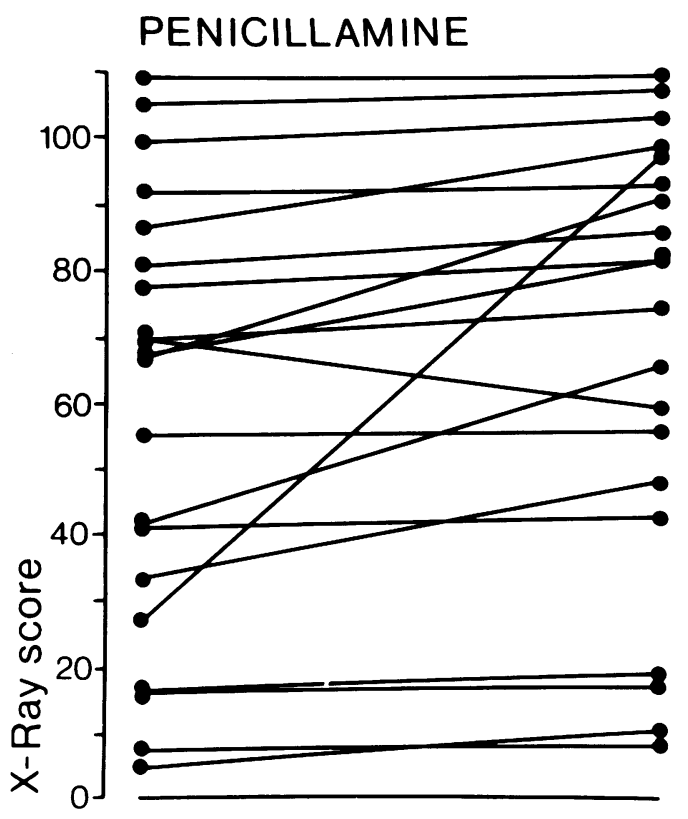

Final

Fig. 3 Change in radiograph score in penicillamine treated group.

gold $=27$ months, penicillamine $=25$ months, and levamisole $=23$ months).

Separate statistical analysis of the hand radiograph scores of each observer showed the following:

(1) The initial scores over the 4 groups were comparable (Kruskal-Wallis test, $\mathrm{p}>0 \cdot 2$ ).

(2) The final scores over the 4 groups were comparable (Kruskal-Wallis test, $\mathrm{p}>0 \cdot 2$ ).

(3) The degree of change in scores in each group was comparable (Kruskal-Wallis test, $\mathrm{p}>0 \cdot 2$ ).

(4) The rate of change in radiograph scores before treatment was comparable in all 4 groups (Kruskal-Wallis test, $\mathrm{p}>0 \cdot 2$ ).

(5) The rate of change over the study period was comparable in all 4 groups (Kruskal-Wallis test $\mathrm{p}>0 \cdot 2 ; t$ test, $\mathrm{p}>0 \cdot 2$ ).

(6) Significant deterioration in score over the period of the study was present in all 4 groups (Wilcoxon test; $\mathrm{p}<0.05 ; t$ test, $\mathrm{p}<0.05$ ).

(7) Rate of erosion was significantly less in all 4 groups compared with the pretreatment rates (Wilcoxon test, $\mathrm{p}<0 \cdot 05$ ).

Change in scores is shown in Figs. 1-4. All groups deteriorated significantly over the study period, with no statistical difference in the rate of deterioration, but the trend favoured a slower rate in gold and penicillamine treated patients. All groups, however, including the control group, showed a slower rate of deterioration during the study as compared with the 


\section{LEVAMISOLE}

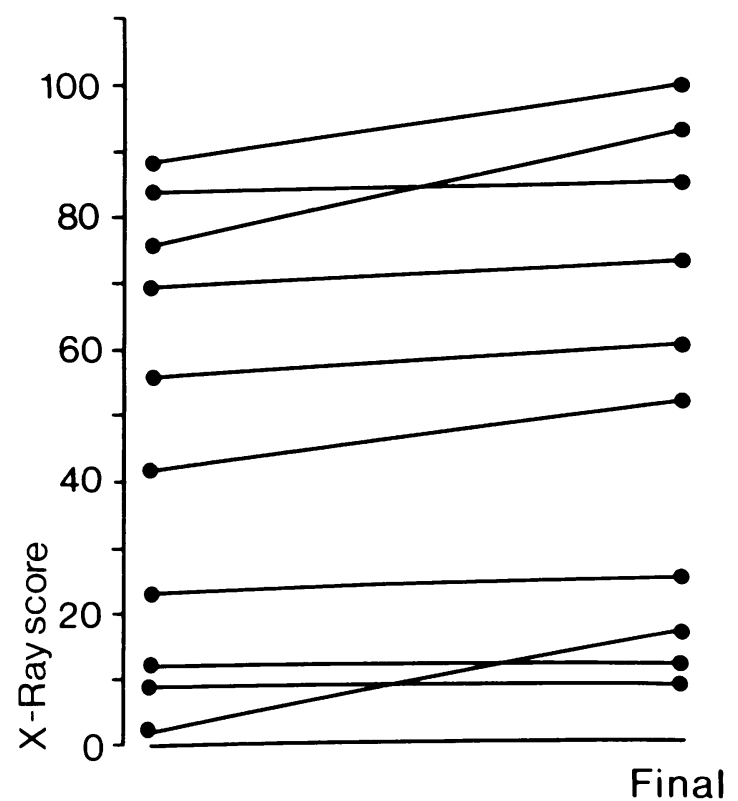

Fig. 4 Change in radiograph score in levamisole treated group.

Table 4 Chronological method (T.P.)

\begin{tabular}{lcll}
\hline $\begin{array}{l}\text { Treatment } \\
\text { group }\end{array}$ & $\begin{array}{l}\text { Correct } \\
\text { order }\end{array}$ & $\begin{array}{l}\text { Discrepant } \\
\text { order }\end{array}$ & $\begin{array}{l}\text { No } \\
\text { difference } \\
\text { in severity }\end{array}$ \\
\hline Control & 8 & 0 & 2 \\
Gold & 18 & 4 & 4 \\
Penicillamine & 13 & 7 & 1 \\
Levamisole & 8 & 0 & 2 \\
\hline
\end{tabular}

$\chi^{2}$ control versus gold $2 \cdot 76$, NS.

$\chi^{2}$ control versus penicillamine $2 \cdot 6$, NS.

$\chi^{2}$ control versus levamisole 0 , NS.

Similar results for H.A.C. and J.A.H.

prestudy rate. The chronological method of assessing hand radiographs showed no significant difference in the number of discrepant results between the control and the treatment groups (Table 4).

\section{Discussion}

Assessment of the possible influence of therapy on radiological change in rhe umatoid arthritis is a difficult task. The few previous studies which have addressed this problem have yielded contradictory results. Sigler et al. ${ }^{3}$ achieved a remarkable 2-year follow-up of 13 placebo treated patients who showed significantly more radiographic changes than 15 patients on intramuscular gold. Our experience of placebo in second-line studies suggests that the patients of Sigler et al. must either have been more tolerant or suffered from milder disease than the patients at our clinic. In view of our knowledge of the symptomatic improvement attributable to secondline the rapy we believe that a similar placebo controlled trial would now be both impracticable and unethical.

In contrast, in an uncontrolled study Gibson et al. ${ }^{7}$ suggested that penicillamine prevented a significant increase in the number of erosions, but they did not find a similar effect with gold. Sharp et al. ${ }^{6}$ in another uncontrolled study showed prevention of radiologically detectable erosive changes by gold in patients who have a clinical response. Luukkainen et al. ${ }^{8}$ showed in their study of intramuscular gold in early disease, greater radiological deterioration in their group on low-dose gold (total $<500 \mathrm{mg}$ ) than in their group on high-dose gold (total $500 \mathrm{mg}$ or more). Unfortunately these groups were not comparable with respect to numbers of erosions at the start of the study.

The methods of assessment described were chosen because of the sensitivity of hand radiographs in following the natural history of erosions in rheumatoid arthritis. The excluded joints of the hands and the joints of the feet may have provided more information, but the state of these joints is difficult to assess because of the frequency of overlapping articular surfaces. We made no attempt to assess joint space narrowing because of the variation in this measurement with joint flexion.

There are a number of possible explanations for failure to demonstrate any significant effect on disease progression. The most obvious is that these drugs have no demonstrable effect on the progress of erosive disease despite altering inflammatory indices.

Another possible explanation is the unsuitability of the control group. Few patients who merit secondline therapy can tolerate a prolonged period without such drugs, and it may be that the patients who can do this are a subgroup with more slowly progressive disease. Nevertheless these patients had ESR and haemoglobin values comparable with those in the treatment groups at the start of the study and failed to show an improvement in these variables over the study period. This suggests that the disease continued to be active in them. (On the other hand, patients on levamisole had no significant changes in ESR or haemoglobin over the period of the study. This may be related to the fact that the initial values in these patients tended to be less abnormal, though they were not statistically significantly different from the 
other groups $(p>0 \cdot 2)$. For these reasons we feel that we can make no comment on the effects of levamisole on radiological progression of the disease, and because of the rapid drop-out rate very large numbers of patients would be required to resolve this question.)

Despite studies by Brook and Corbett ${ }^{12}$ little is known about the expected rate of bone destruction in a group of rheumatoid patients with disease of sufficient severity to merit second-line drugs.

It is likely that larger numbers of patients would be required to show a significant change in radiological progression in a comparison between groups of treated and untreated patients (though our numbers are similar to those in other studies). During the period of study the mean deterioration in radiograph scores was: control, $12 \cdot 5$; gold, 8.6 ; penicillamine, $9 \cdot 2$. Given these changes in scores and using the formula

$$
\mathrm{n}=(\mathrm{SD})^{2} \frac{(\alpha+\beta)^{2}}{\text { diff }^{2}}
$$

(where $\mathrm{n}=$ number of patients in each group, $\alpha=$ type I error probability of $5 \%, \beta=$ type II error probability of $20 \%$ (i.e., $80 \%$ power), diff $=$ difference between radiograph score change in 2 groups).

We would require 120 patients remaining on therapy for 2 years in each group to show a significant difference between control and gold groups and 161 patients in each group to show a significant difference between control and penicillamine groups. Since there is a high drop-out rate on both gold and penicillamine between 0 and 2 years, 3 or 4 times this number would have to be enrolled to obtain a valid number.

Conversely, with the number in our study we would have required a difference of over 108 between the change in the control and treatment radiograph scores to demonstrate a significantly beneficial drug effect. However, we found a difference of only $3 \cdot 3$ between gold and control groups and only $3 \cdot 3$ between control and penicillamine groups.

We used a number of different statistical methods in this study in an attempt to reproduce the statistical methods used by other authors. Rate of erosion was assessed, as this has been used in at least one previous study ${ }^{13}$ which reported a significant effect. Using this method on our results we found a significant slowing of erosive disease in all groups, including control. As this method relies on the unproved assumption that erosive disease progresses in a linear fashion, we would advise extreme caution in interpreting rate results.

Controls were followed up for longer periods than those on active drugs; thus we have to some extent favoured the active drug in our assessment. Despite the failure to demonstrate significant radiological improvement there was a favourable trend with both gold and penicillamine, and we still advocate the use of those 2 drugs in active rhe umatoid arthritis in view of the clinical benefit which can follow.

An important aim of second-line therapy may be the limitation of the need for joint surgery. The data in this paper are gathered from joints which are less amenable than some to joint replacement, and there is no indication that the degree of destruction in these small joints correlates with the need for surgical intervention elsewhere.

There has been much discussion about the use of second-line drugs in early rather than more advanced disease. Early, mild disease might simplify the counting of erosions, but in practice an active inflammatory component may respond to second-line drugs at many stages of the progression of rheumatoid arthritis. Any realistic assessment of this therapy must take cognisance of this fact despite the technical problems encountered.

This study has attempted to resolve the difficulties of radiographic assessment of second-line drugs in rheumatoid arthritis. The control group selected may not have been a true placebo but was, we felt, the best that could reasonably be achieved. No significant radiological effect of second-line therapy could be demonstrated despite a favourable trend and a clear clinical and haematological response. Is radiological monitoring of second-line therapy, therefore, an unattainable goal? Much time and effort is spent on such monitoring. The results of this study, using the drugs and radiographic methods available, lead to the conclusion that the number of patients at a single centre cannot provide a decisive answer. Even with multicentre co-operation, which is difficult to achieve, the problem of a control group appears insurmountable. A rheumatological impasse?

We thank Robert Murdoch and David Hole for statistical and computing help and Maureen Tucker for typing the manuscript.

\section{References}

1 Andrews F M, Golding D N, Freeman A M, et al. Controlled trial of $\mathrm{D}(-)$ penicillamine in severe rheumatoid arthritis. Lancet 1973; i: 275-80.

2 Srinivasan R, Miller B L, Paulus H E. Long-term chrysatherapy in rheumatoid arthritis. Arthritis Rheum 1979; 22: 105-10.

3 Sigler J W, Bluhm G B, Duncan H, et al. Gold salts in the treatment of rheumatoid arthritis. Ann Intern Med 1974; 80: 21-6.

4 Rothermich N O, Thomas M H, Phillips U K, Bergen W. Clinical trial of penicillamine in rheumatoid arthritis. Arthritis Rheum 1981; 24: 1473-8.

5 Day A T, Golding $J \mathbf{R}$, Lee $P \mathrm{~N}$, Butterworth A $D$. Penicillamine in rheumatoid disease: a long term study. $\mathrm{Br}$ Med J 1974; i: 180-3. 
6 Sharp J T, Lidsky M D, Duffy J. Clinical responses during gold therapy for rheumatoid arthritis. Arthritis Rheum 1982; 25: 540-9.

7 Gibson T, Huskisson E C, Wojtulewski J A, et al. Evidence that D-penicillamine alters the course of rheumatoid arthritis. Rheumatol Rehabil 1976; 15: 211-5.

8 Luukkainen R, Isomaki H, Kajander A. Effect of gold on the progression of erosions in RA patients. Scand $J$ Rheumatol 1977; 6: 123-7.

9 Pullar T, Hunter J A, Capell H A. Gold and penicillamine therapy: is shared care with general practitioners effective and safe? Rheumatol Rehabil 1982; 21 : 139-44.
10 Capell H A, Hunter J A, Rennie J A N, Murdoch R M. Levamisole-a possible alternative to gold and penicillamine in the long-term treatment of rheumatoid arthritis? J Rheumatol $1981 ; 8: 730-40$.

11 Sharp J T, Lidsky M D, Collins L C, Moreland J. Methods of scoring the progression of radiological changes in rheumatoid arthritis. Arthritis Rheum 1971; 14: 706-20.

12 Brook A, Corbett M. Radiographic changes in early rheumatoid disease. Ann Rheum Dis 1977; 36: 71-3.

13 Bluhm G B, Smith D W, Mikulaschek W M. Radiological assessment of benoxaprofen therapy in rheumatoid arthritis. Eur J Rheumatol Inflamm 1982; 5: 186-97.

\section{Book review}

Locomotor Disability in General Practice. Oxford General Practice Series 5. Eds. Malcolm I. V. Jayson and Raymond Million. Pp. 347.£12·50. Oxford University Press: Oxford. 1983.

A series of books designed specifically to look at areas of general practice and help the general practitioner in his management has been long overdue. This volume on locomotor problems is the fifth of the series and is jointly edited by a general practitioner and a rheumatologist. It has 18 contributors, four of whom are general practitioners, and is designed to cover a large and important area of the general practitioner's workload.

Although there are a number of valuable and interesting chapters, the book is uneven and lacks a cohesive thread. The opening section on 'General Topics' begins with an excellent chapter on the epidemiology of locomotor disorders in general practice and includes chapters on aches and pains, psychological aspects of rheumatoid arthritis, and back pain and peripheral vascular disorders. The problem of combining a 'systems' as well as 'symptoms' approach continues in later chapters. Some contributors have attempted to cram too much into individual chapters, with loss of clarity, and many of the charts and tables are difficult to read and contain too much detail.
The chapter on back pain and sciatica is well laid out and clearly presented with a practical approach and an emphasis on management by the general practitioner. In contradistinction, however, the chapter on disorders of hand function from an orthopaedic viewpoint has little relevance to management by a general practitioner. At the end of the book there are two valuable chapters on the management of common handicaps and the management of rheumatic disease, and perhaps greater emphasis could have been made throughout the book on enabling the GP to manage common problems in his own surgery.

Reference is made in the chapter on health care teams to the high level of referral for rheumatic problems. This is probably related not only to the fact that rheumatology has developed after many GPs qualified but also to the availability of direct referral for splints, appliances, and physiotherapy. I am sure the practice physiotherapist mentioned is a very rare bird indeed.

It is surprising that the only paramedical contribution is by a chiropodist and neither physiotherapy nor occupational therapy are represented.

This book is a useful addition to a general practice library, and, hopefully, future editions will improve on format and relevance. 